\title{
Mixed-Type Reverse Order Laws for Generalized Inverses over Hilbert Space
}

\author{
Haiyan Zhang, Fengling Lu \\ College of Mathematics and Satistics, Shangqiu Normal University, Shangqiu, China \\ Email: csqam@163.com
}

How to cite this paper: Zhang, H.Y. and Lu, F.L. (2017) Mixed-Type Reverse Order Laws for Generalized Inverses over Hilbert Space. Applied Mathematics, 8, 637-644. https://doi.org/10.4236/am.2017.85050

Received: April 4, 2017

Accepted: May 19, 2017

Published: May 22, 2017

Copyright $\odot 2017$ by authors and Scientific Research Publishing Inc. This work is licensed under the Creative Commons Attribution International License (CC BY 4.0).

http://creativecommons.org/licenses/by/4.0/

\section{(c) (i) Open Access}

\section{Abstract}

In this paper, by using a block-operator matrix technique, we study mixed-type reverse order laws for $\{1,3\}-,\{1,2,3\}$ - and $\{1,3,4\}$-generalized inverses over Hilbert spaces. It is shown that $\left\{(A B)^{(1,3)}\right\}=\left\{B^{(1,3)}\left(A B B^{(1,3)}\right)^{(1,3)}\right\}$ and $\left\{(A B)^{(1,2, i)}\right\}=\left\{B^{(1,2, i)}\left(A B B^{(1,2, i)}\right)^{(1,2, i)}\right\},(i=3,4)$ when the ranges of $A, B, A B$ are closed. Moreover, a new equivalent condition of $\left\{(A B)^{(1,3,4)}\right\}=$ $\left\{B^{(1,3,4)}\left(A B B^{(1,3,4)}\right)^{(1,3,4)}\right\}$ is given.

\section{Keywords}

$\{1,2,3\}$-Reverse, $\{1,3,4\}$-Reverse, Reverse Order Law, Block-Operator Matrix

\section{Introduction}

The reverse order law of generalized inverses plays an important role in theoretic research and numerical computations in many areas, including the singular matrix problem and optimization problem. They have attracted considerable attention since the middle 1960s, and many interesting results have been studied, see [1]-[10].

For convenience, we firstly introduce some notations. Let $H$ and $K$ be infinite dimensional Hilbert spaces and $B(H, K)$ be the set of all bounded linear operators from $H$ to $K$ and abbreviate $B(K, H)$ to $B(H)$ if $K=H$. For an operator $A \in B(H, K), N(A)$ and $R(A)$ are the null space and the range of $A$, respectively. Denote by $A^{*}$ the adjoint of $A$. Recall that $A \in B(H, K)$ has a Moore-Penrose inverse if there exists an operator $G \in B(K, H)$ satisfies the following four equations, which is said to be the Moore-Penrose conditions: 


$$
\text { (1) } A G A=A \text {; (2) } G A G=G ;(3)(A G)^{*}=A G ;(4)(G A)^{*}=G A \text {. }
$$

If one exists, the Moore-Penrose inverse of $A$ is unique and it is denoted by $A^{+}$. And let $A\{i, j, \cdots, k\}$ denote the set of all operator $G \in B(K, H)$ which satisfy equations $(i),(j), \cdots,(k)$ from among the above Moore-Penrose equations. Such $G$ will be called a $\{i, j, k\}$-inverse of $A$ and will be denoted by $A^{(i, j, k)}$. evidently, $A\{1,2,3,4\}=A^{+}$when $A$ has closed range.

For the Moore-Penrose inverse, Greville [2] gave the necessary and sufficient conditions for $(A B)^{+}=B^{+} A^{+}$on matrix algebra, this result was extended to bounded operators on Hilbert space by Izumino [4]. Subsequently, some researcher discussed the reverse order laws of other type generalized inverses, such as $(A B) \theta=B \theta A \theta, \theta \subset\{1,2,3,4\}$ [5] [6] [8] [10]. The mixed-type reverse-order laws for $A B$ like $(A B)^{+}=B^{+}\left(A^{+} A B\right)^{+}$and $(A B)^{+}=\left(A^{+} A B\right)^{+} A^{+}$were considered in [3] [4] when $A$ and $B$ are matrices. Motivated by this, Wang et al. [7] studied the mixed-type reverse-order laws for $A B^{(1,3)}$. Yang and Liu [9] gave the equivalent condition of $\left\{(A B)^{(1,2, i)}\right\}=\left\{B^{(1,2, i)}\left(A B B^{(1,2, i)}\right)^{(1,2, i)}\right\},(i=3,4)$, by using the extremal ranks of generalized Schur complements, when $A$ and $B$ are matrices. The mixed-type reverse order laws of $(A B)^{(1,3,4)}$ were discussed on operator space over Hilbert space [5].

In this article, we study the mixed-type reverse order laws of $(A B)^{(1,2, i)}$, $(A B)^{(1,3)}$ and $(A B)^{(1,3,4)}$ over infinite Hilbert space by using a block-operator matrix technique. For given $A, B$, it is shown that

$$
\left\{(A B)^{(1,3)}\right\}=\left\{B^{(1,3)}\left(A B B^{(1,3)}\right)^{(1,3)}\right\}
$$

and

$$
\left\{(A B)^{(1,2, i)}\right\}=\left\{B^{(1,2, i)}\left(A B B^{(1,2, i)}\right)^{(1,2, i)}\right\},(i=3,4)
$$

when the ranges of $A, B, A B$ are closed. We generalized the results from [7] and [9] to the case of bounded linear operators on Hilbert spaces. Moreover, a new equivalent condition of $\left\{(A B)^{(1,3,4)}\right\}=\left\{B^{(1,3,4)}\left(A B B^{(1,3,4)}\right)^{(1,3,4)}\right\}$ is given.

\section{Main Results}

To obtain our main results, we begin with some notations and lemmas. Let $A \in B(H, K)$ with closed range. It is well known that $A$ has the following matrix decomposition

$$
A=\left[\begin{array}{cc}
A_{1} & 0 \\
0 & 0
\end{array}\right]:\left[\begin{array}{c}
R\left(A^{*}\right) \\
N(A)
\end{array}\right] \rightarrow\left[\begin{array}{c}
R(A) \\
N\left(A^{*}\right)
\end{array}\right],
$$

where $A_{1}$ is invertible. Also, $A^{+}$has the form

$$
A^{+}=\left[\begin{array}{cc}
A_{1}^{-1} & 0 \\
0 & 0
\end{array}\right]:\left[\begin{array}{c}
R(A) \\
N\left(A^{*}\right)
\end{array}\right] \rightarrow\left[\begin{array}{c}
R\left(A^{*}\right) \\
N(A)
\end{array}\right] .
$$


The $\{1,2,3\}$ - and $\{1,3,4\}$-inverse has also similarly matrix form.

Lemma 1 ([5]). Let $A \in B(H, K)$ have closed range. Then

$$
\begin{aligned}
& A\{1,3\}=\left\{\left[\begin{array}{cc}
A_{1}^{-1} & 0 \\
X_{3} & X_{4}
\end{array}\right]:\left[\begin{array}{c}
R(A) \\
N\left(A^{*}\right)
\end{array}\right] \rightarrow\left[\begin{array}{c}
R\left(A^{*}\right) \\
N(A)
\end{array}\right]\right\}, \\
& A\{1,2,3\}=\left\{\left[\begin{array}{cc}
A_{1}^{-1} & 0 \\
X_{3} & 0
\end{array}\right]:\left[\begin{array}{c}
R(A) \\
N\left(A^{*}\right)
\end{array}\right] \rightarrow\left[\begin{array}{c}
R\left(A^{*}\right) \\
N(A)
\end{array}\right]\right\}
\end{aligned}
$$

and

$$
A\{1,3,4\}=\left\{\left[\begin{array}{cc}
A_{1}^{-1} & 0 \\
0 & X_{4}
\end{array}\right]:\left[\begin{array}{c}
R(A) \\
N\left(A^{*}\right)
\end{array}\right] \rightarrow\left[\begin{array}{c}
R\left(A^{*}\right) \\
N(A)
\end{array}\right]\right\} .
$$

Let $A \in B(H, K), \quad B \in B(L, H)$ and $A B \in B(L, K)$ with closed ranges. For convenience, denote by

$$
\left\{\begin{array} { l } 
{ H _ { 1 } = R ( B ) \cap N ( A ) , } \\
{ H _ { 2 } = R ( B ) \Theta ^ { \perp } H _ { 1 } , } \\
{ H _ { 3 } = N ( B ^ { * } ) \cap N ( A ) , } \\
{ H _ { 4 } = N ( B ^ { * } ) \Theta ^ { \perp } H _ { 3 } , }
\end{array} \quad \left\{\begin{array}{l}
K_{1}=R(A B) \\
K_{2}=R(A) \Theta^{\perp} R(A B)
\end{array},\right.\right.
$$

and

$$
L_{1}=B^{+}\left(H_{1}\right), L_{2}=R\left(B^{*}\right) \Theta^{\perp} L_{1}
$$

then

$$
H=H_{1} \oplus H_{2} \oplus H_{3} \oplus H_{4}, \quad K=K_{1} \oplus K_{2} \oplus N\left(A^{*}\right)
$$

and

$$
L=L_{1} \oplus L_{2} \oplus N(B) .
$$

Under these space decomposition, we get two useful representations of operators $A \in B(H, K)$ and $B \in B(L, K)$.

Lemma 2 ([9] [10]). Let $A \in B(H, K), B \in B(L, K)$ such that $R(A), R(B)$ and $R(A B)$ are closed.

If $A B \neq 0$, the following statements hold,

(1) When $H_{1} \neq\{0\}, A$ and $B$ have the matrix form as follows, respectively,

$$
\begin{aligned}
A & =\left[\begin{array}{cccc}
0 & A_{12} & 0 & A_{14} \\
0 & 0 & 0 & A_{24} \\
0 & 0 & 0 & 0
\end{array}\right]:\left[\begin{array}{c}
H_{1} \\
H_{2} \\
H_{3} \\
H_{4}
\end{array}\right] \rightarrow\left[\begin{array}{c}
K_{1} \\
K_{2} \\
N\left(A^{*}\right)
\end{array}\right], \\
B & =\left[\begin{array}{ccc}
B_{11} & B_{12} & 0 \\
0 & B_{22} & 0 \\
0 & 0 & 0 \\
0 & 0 & 0
\end{array}\right]:\left[\begin{array}{c}
L_{1} \\
L_{2} \\
N(B)
\end{array}\right] \rightarrow\left[\begin{array}{c}
H_{1} \\
H_{2} \\
H_{3} \\
H_{4}
\end{array}\right],
\end{aligned}
$$

where $A_{11}, B_{11}, B_{22}$ are invertible and $A_{22}$ is surjective.

(2) When $H_{1}=\{0\}$, 


$$
\begin{aligned}
& A=\left[\begin{array}{ccc}
A_{12} & 0 & A_{14} \\
0 & 0 & A_{24} \\
0 & 0 & 0
\end{array}\right]:\left[\begin{array}{l}
H_{2} \\
H_{3} \\
H_{4}
\end{array}\right] \rightarrow\left[\begin{array}{c}
K_{1} \\
K_{2} \\
N\left(A^{*}\right)
\end{array}\right], \\
& B=\left[\begin{array}{cc}
B_{22} & 0 \\
0 & 0 \\
0 & 0
\end{array}\right]:\left[\begin{array}{c}
L_{2} \\
N(B)
\end{array}\right] \rightarrow\left[\begin{array}{c}
H_{2} \\
H_{3} \\
H_{4}
\end{array}\right],
\end{aligned}
$$

where $A_{12}, B_{22}$ are invertible and $A_{24}$ is surjective.

Theorem 3. Let $A \in B(H, K), B \in B(L, H)$ such that $R(A), R(B)$ and $R(A B)$ are closed. Then

$$
\left\{(A B)^{(1,2,3)}\right\}=\left\{B^{(1,2,3)}\left(A B B^{(1,2,3)}\right)^{(1,2,3)}\right\} .
$$

Proof If $A B=0$, then $(A B)\{1,2,3\}=\{0\}$, the result holds. So assume that $A B \neq 0$. Next, we divide the proof into two cases.

Case 1. $H_{1} \neq\{0\}$.

In this case, $A, B$ have matrix forms (2.3) and (2.4), respectively. This implies that

$$
A B=\left[\begin{array}{ccc}
0 & A_{12} B_{22} & 0 \\
0 & 0 & 0 \\
0 & 0 & 0
\end{array}\right]:\left[\begin{array}{c}
J_{1} \\
J_{2} \\
N(B)
\end{array}\right] \rightarrow\left[\begin{array}{c}
K_{1} \\
K_{2} \\
N\left(A^{*}\right)
\end{array}\right] .
$$

Using Lemma 1, we get

$$
B^{(123)}=\left[\begin{array}{cccc}
B_{11}^{-1} & -B_{11}^{-1} B_{12} B_{22}^{-1} & 0 & 0 \\
0 & B_{22}^{-1} & 0 & 0 \\
F_{31} & F_{32} & 0 & 0
\end{array}\right]:\left[\begin{array}{c}
H_{1} \\
H_{2} \\
H_{3} \\
H_{4}
\end{array}\right] \rightarrow\left[\begin{array}{c}
L_{1} \\
L_{2} \\
N(B)
\end{array}\right],
$$

and

$$
(A B)^{(123)}=\left[\begin{array}{ccc}
M_{11} & 0 & 0 \\
B_{22}^{-1} A_{12}^{-1} & 0 & 0 \\
M_{31} & 0 & 0
\end{array}\right]:\left[\begin{array}{c}
K_{1} \\
K_{2} \\
N\left(A^{*}\right)
\end{array}\right] \rightarrow\left[\begin{array}{c}
L_{1} \\
L_{2} \\
N(B)
\end{array}\right] .
$$

where $F_{31}, F_{32}, M_{11}, M_{31}$ are arbitrary. Combining formulae (2.7) with (2.8), it is easy to get

$$
A B B^{(123)}=\left[\begin{array}{cccc}
0 & A_{12} & 0 & 0 \\
0 & 0 & 0 & 0 \\
0 & 0 & 0 & 0
\end{array}\right]:\left[\begin{array}{c}
H_{1} \\
H_{2} \\
H_{3} \\
H_{4}
\end{array}\right] \rightarrow\left[\begin{array}{c}
K_{1} \\
K_{2} \\
N\left(A^{*}\right)
\end{array}\right] .
$$

Using Lemma 1 again, we have

$$
\left(A B B^{(123)}\right)^{(123)}=\left[\begin{array}{lll}
G_{11} & 0 & 0 \\
A_{12}^{-1} & 0 & 0 \\
G_{31} & 0 & 0 \\
G_{41} & 0 & 0
\end{array}\right]:\left[\begin{array}{c}
K_{1} \\
K_{2} \\
N\left(A^{*}\right)
\end{array}\right] \rightarrow\left[\begin{array}{c}
H_{1} \\
H_{2} \\
H_{3} \\
H_{4}
\end{array}\right],
$$


where $G_{i 1}, i=1,3,4$ are arbitrary. By direct computation, it is clearly from (2.8) and (2.10) that

$$
B^{(123)}\left(A B B^{(123)}\right)^{(123)}=\left[\begin{array}{ccc}
P_{11} & 0 & 0 \\
B_{22}^{-1} & 0 & 0 \\
P_{31} & 0 & 0
\end{array}\right]:\left[\begin{array}{c}
K_{1} \\
K_{2} \\
N\left(A^{*}\right)
\end{array}\right] \rightarrow\left[\begin{array}{c}
L_{1} \\
L_{2} \\
N(B)
\end{array}\right],
$$

where $P_{11}=B_{11}^{-1} G_{11}-B_{11}^{-1} B_{12} B_{22}^{-1} A_{12}^{-1}, \quad P_{31}=F_{31} G_{11}+F_{32} A_{12}^{-1}$. Thus, by the arbitrariness of $G_{11}, F_{31}, F_{32}$, it follows from fromulae (2.9) and (2.11) that

$$
\left\{(A B)^{(1,2,3)}\right\}=\left\{B^{(1,2,3)}\left(A B B^{(1,2,3)}\right)^{(1,2,3)}\right\}
$$

holds.

Case $2 H_{1}=\{0\}$. Obviously, $L_{1}=\{0\}$. Consequently, $H=H_{2} \oplus H_{3} \oplus H_{4}$ and $L=L_{2} \oplus N(B)$. By Lemma 2, A,B have matrix forms (2.5) and (2.6), respectively. This follows that

$$
A B=\left[\begin{array}{cc}
A_{12} B_{22} & 0 \\
0 & 0 \\
0 & 0
\end{array}\right]:\left[\begin{array}{c}
L_{2} \\
N(B)
\end{array}\right] \rightarrow\left[\begin{array}{c}
K_{1} \\
K_{2} \\
N\left(A^{*}\right)
\end{array}\right] .
$$

By Lemma 1, we get

$$
B^{(123)}=\left[\begin{array}{lll}
B_{22}^{-1} & 0 & 0 \\
F_{21} & 0 & 0
\end{array}\right]:\left[\begin{array}{l}
H_{2} \\
H_{3} \\
H_{4}
\end{array}\right] \rightarrow\left[\begin{array}{c}
L_{2} \\
N(B)
\end{array}\right],
$$

and

$$
(A B)^{(123)}=\left[\begin{array}{ccc}
B_{22}^{-1} A_{12}^{-1} & 0 & 0 \\
M_{21} & 0 & 0
\end{array}\right]:\left[\begin{array}{c}
K_{1} \\
K_{2} \\
N\left(A^{*}\right)
\end{array}\right] \rightarrow\left[\begin{array}{c}
L_{2} \\
N(B)
\end{array}\right] .
$$

where $F_{21}, M_{21}$ are arbitrary. Combining formulae (2.12) with (2.13),

$$
A B B^{(123)}=\left[\begin{array}{ccc}
A_{12} & 0 & 0 \\
0 & 0 & 0 \\
0 & 0 & 0
\end{array}\right]:\left[\begin{array}{c}
H_{2} \\
H_{3} \\
H_{4}
\end{array}\right] \rightarrow\left[\begin{array}{c}
K_{1} \\
K_{2} \\
N\left(A^{*}\right)
\end{array}\right] .
$$

Again from Lemma 1,

$$
\left(A B B^{(123)}\right)^{(123)}=\left[\begin{array}{lll}
A_{12}^{-1} & 0 & 0 \\
Q_{21} & 0 & 0 \\
Q_{31} & 0 & 0
\end{array}\right]:\left[\begin{array}{c}
K_{1} \\
K_{2} \\
N\left(A^{*}\right)
\end{array}\right] \rightarrow\left[\begin{array}{c}
H_{2} \\
H_{3} \\
H_{4}
\end{array}\right],
$$

where $Q_{21}, Q_{31}$ are arbitrary. By direct computation, it is clearly that

$$
B^{(123)}\left(A B B^{(123)}\right)^{(123)}=\left[\begin{array}{lll}
B_{22}^{-1} A_{12}^{-1} & 0 & 0 \\
F_{21} A_{12}^{-1} & 0 & 0
\end{array}\right]:\left[\begin{array}{c}
K_{1} \\
K_{2} \\
N\left(A^{*}\right)
\end{array}\right] \rightarrow\left[\begin{array}{c}
L_{2} \\
N(B)
\end{array}\right] .
$$


Thus, from fromulae (2.14) and (2.15), it is clear that

$$
\left\{(A B)^{(1,2,3)}\right\}=\left\{B^{(1,2,3)}\left(A B B^{(1,2,3)}\right)^{(1,2,3)}\right\}
$$

also holds in this case. The proof is completed.

From the relationship of $\{1,2,3\}$-inverse and $\{1,2,4\}$-inverse, we can obtain the following result without proof.

Corollary 4. Let $A \in B(H, K), B \in B(L, H)$ such that $R(A), R(B)$ and $R(A B)$ are closed. Then

$$
\left\{(A B)^{(1,2,4)}\right\}=\left\{B^{(1,2,4)}\left(A B B^{(1,2,4)}\right)^{(1,2,4)}\right\} .
$$

Similar to the proof of Theorem 3, we also can get the following result.

Theorem 5. Let $A \in B(H, K), B \in B(L, H)$ such that $R(A), R(B)$ and $R(A B)$ are closed. Then

$$
\left\{(A B)^{(1,3)}\right\}=\left\{B^{(1,3)}\left(A B B^{(1,3)}\right)^{(1,3)}\right\} .
$$

In [5], the author gave a necessary and sufficient condition of $\left\{(A B)^{(1,3,4)}\right\}=\left\{B^{(1,3,4)}\left(A B B^{(1,3,4)}\right)^{(1,3,4)}\right\}$. Next, we give a new equivalent condition of the mixed-type reverse order law for $(A B)^{(134)}$.

Theorem 6. Let $A \in B(H, K), B \in B(L, H)$ such that $R(A), R(B)$ and $R(A B)$ are closed. If $A B \neq 0$, the following statements are equivalent,

(1) $\left\{(A B)^{(1,3,4)}\right\}=\left\{B^{(1,3,4)}\left(A B B^{(1,3,4)}\right)^{(1,3,4)}\right\}$;

(2) $R\left(B B^{*} A^{*}\right) \subset R\left(A^{*}\right)$.

Proof We divide the proof into two cases.

Case 1. $H_{1} \neq\{0\}$. Using Lemma 2, $A, B$ have matrix forms (2.3) and (2.4), respectively. The operator $A B$ has the matrix decomposition (2.7). Then

$$
A^{*}=\left[\begin{array}{ccc}
0 & 0 & 0 \\
A_{12}^{*} & 0 & 0 \\
0 & 0 & 0 \\
A_{14}^{*} & A_{24}^{*} & 0
\end{array}\right]:\left[\begin{array}{c}
K_{1} \\
K_{2} \\
N\left(A^{*}\right)
\end{array}\right] \rightarrow\left[\begin{array}{c}
H_{1} \\
H_{2} \\
H_{3} \\
H_{4}
\end{array}\right]
$$

and

$$
B B^{*} A^{*}=\left[\begin{array}{ccc}
B_{12} B_{12}^{*} A_{12}^{*} & 0 & 0 \\
B_{22} B_{12}^{*} A_{12}^{*} & 0 & 0 \\
0 & 0 & 0 \\
0 & 0 & 0
\end{array}\right]:\left[\begin{array}{c}
K_{1} \\
K_{2} \\
N\left(A^{*}\right)
\end{array}\right] \rightarrow\left[\begin{array}{c}
H_{1} \\
H_{2} \\
H_{3} \\
H_{4}
\end{array}\right]
$$

by direct computation from (2.3) and (2.4). Therefore, comparing (2.16) with (2.17), it is natural that $R\left(B B^{*} A^{*}\right) \subset R\left(A^{*}\right)$ if and only if $B_{12} B_{12}^{*} A_{12}^{*}=0$. So $R\left(B B^{*} A^{*}\right) \subset R\left(A^{*}\right)$ if and only if $B_{12}=0$ since $A_{12}$ is invertilbe.

On the other hand, it follows from Lemma 1 that 


$$
B^{(134)}=\left[\begin{array}{cccc}
B_{11}^{-1} & -B_{11}^{-1} B_{12} B_{22}^{-1} & 0 & 0 \\
0 & B_{22}^{-1} & 0 & 0 \\
0 & 0 & F_{33} & F_{34}
\end{array}\right]:\left[\begin{array}{c}
H_{1} \\
H_{2} \\
H_{3} \\
H_{4}
\end{array}\right] \rightarrow\left[\begin{array}{c}
L_{1} \\
L_{2} \\
N(B)
\end{array}\right],
$$

and

$$
(A B)^{(134)}=\left[\begin{array}{ccc}
0 & M_{12} & M_{13} \\
B_{22}^{-1} A_{12}^{-1} & 0 & 0 \\
0 & M_{32} & M_{33}
\end{array}\right]:\left[\begin{array}{c}
K_{1} \\
K_{2} \\
N\left(A^{*}\right)
\end{array}\right] \rightarrow\left[\begin{array}{c}
L_{1} \\
L_{2} \\
N(B)
\end{array}\right] .
$$

where $F_{33}, F_{34}, M_{i j},(i=1,3, j=2,3)$ are arbitrary.

Combining formulae (2.7) with (2.18), it is easy to get

$$
A B B^{(134)}=\left[\begin{array}{cccc}
0 & A_{12} & 0 & 0 \\
0 & 0 & 0 & 0 \\
0 & 0 & 0 & 0
\end{array}\right]:\left[\begin{array}{c}
H_{1} \\
H_{2} \\
H_{3} \\
H_{4}
\end{array}\right] \rightarrow\left[\begin{array}{c}
K_{1} \\
K_{2} \\
N\left(A^{*}\right)
\end{array}\right] .
$$

Using Lemma 1 again, we have

$$
\left(A B B^{(134)}\right)^{(134)}=\left[\begin{array}{ccc}
0 & G_{12} & G_{13} \\
A_{12}^{-1} & 0 & 0 \\
0 & G_{32} & G_{33} \\
0 & G_{42} & G_{43}
\end{array}\right]:\left[\begin{array}{c}
K_{1} \\
K_{2} \\
N\left(A^{*}\right)
\end{array}\right] \rightarrow\left[\begin{array}{c}
H_{1} \\
H_{2} \\
H_{3} \\
H_{4}
\end{array}\right] .
$$

where $G_{i j}(i=1,3,4, j=2,3)$ are arbitrary. By direct computation, it is clearly from (2.18) and (2.20) that

$$
B^{(134)}\left(A B B^{(134)}\right)^{(134)}=\left[\begin{array}{ccc}
-B_{11}^{-1} B_{12} B_{22}^{-1} A_{12}^{-1} & B_{11}^{-1} G_{12} & B_{11}^{-1} G_{13} \\
B_{22}^{-1} A_{12}^{-1} & 0 & 0 \\
0 & F_{33} G_{32}+F_{34} G_{42} & F_{33} G_{33}+F_{34} G_{43}
\end{array}\right] \text {. }
$$

Comparing (2.21) with (2.19), we have

$$
\left\{(A B)^{(1,3,4)}\right\}=\left\{B^{(1,3,4)}\left(A B B^{(1,3,4)}\right)^{(1,3,4)}\right\}
$$

if and only if $-B_{11}^{-1} B_{12} B_{22}^{-1} A_{12}^{-1}=0$, that is, $B_{12}=0$. Therefore, $\left\{(A B)^{(1,3,4)}\right\}=$ $\left\{B^{(1,3,4)}\left(A B B^{(1,3,4)}\right)^{(1,3,4)}\right\}$ if and only if $R\left(B B^{*} A^{*}\right) \subset R\left(A^{*}\right)$.

Case $2 H_{1}=\{0\}$. Then $A, B$ have matrix forms (2.5) and (2.6), respectively. By similarly discussing to case 1 and case 2 in the proof of Theorem 3.2, it is easy to get that $\left\{(A B)^{(1,3,4)}\right\}=\left\{B^{(1,3,4)}\left(A B B^{(1,3,4)}\right)^{(1,3,4)}\right\}$ and $R\left(B B^{*} A^{*}\right) \subset R\left(A^{*}\right)$ always hold in this case.

So the proof is completed.

\section{Acknowledgements}

This subject is supported by NSF of China (No. 11501345) and the Natural Science Basic Research Plan of Henan Province (No. 152300410221). 


\section{References}

[1] Cvetković-Ilić, D.S. and Djikić, M. (2016) Various Solutions to Reverse Order Law Problems. Linear and Multilinear Algebra, 64, 1207-1219. https://doi.org/10.1080/03081087.2015.1082956

[2] Greville, T.N.E. (1966) Note on the Generalized Inverse of a Matrix Product. Society for Industrial and Applied Mathematics Review, 8, 518-521. https://doi.org/10.1137/1008107

[3] Galperin, A.M. and Waksman, Z. (1980) On Pseudo Inverse of Operator Products. Linear Algebra and Applications, 33, 123-131.

[4] Izumino, S. (1982) The Product of Operators with Closed Range and an Extension of the Reverse Order Law. Tohoku Mathematical Journal, 34, 43-52. https://doi.org/10.2748/tmj/1178229307

[5] Liu, X.J., Huang, S. and Cvetković-Ilić, D.S. (2012) Mixed-Type Reverse-Order Laws for $\{1,3,4\}$-Generalized Inverses over Hilbert Spaces. Applied Mathematics and Computation, 218, 8570-8577.

[6] Liu, X.J., Wu, S.X. and Cvetković-Ilić, D.S. (2013) New Results on Reverse Order Law for $\{1,2,3\}$ - and $\{1,2,4\}$-Inverses of Bounded Operators. Mathematics of Computation, 82, 1597-1607. https://doi.org/10.1090/S0025-5718-2013-02660-9

[7] Wang, M., Wei, M. and Jia, Z. (2009) Mixed-Type Reverse-Order Law of $(A B)^{(13)}$. Linear Algebra and Applications, 430, 1691-1699.

[8] Wang, J., Zhang, H.Y. and Ji, G.X. (2010) A Generalized Reverse Order Law for the Products of Two Operators. Journal of Shaanxi Normal University (Natural Science), 38, 13-17.

[9] Yang, H. and Lu, X.F. (2011) Mixed-Type Reverse-Order Laws of $(A B)^{(123)}$ and $(A B)^{(124)}$. Applied Mathematics and Computation, 217, 10361-10367. https://doi.org/10.1016/j.amc.2011.05.046

[10] Zhang, H.Y. and Si, H.Y. (2016) Reverse Order Laws for $\{1,2,3\}$ - Inverse of Two-Operator Product. Journal of Sichuan Normal University (Natural Science), 39, 671-677.

Submit or recommend next manuscript to SCIRP and we will provide best service for you:

Accepting pre-submission inquiries through Email, Facebook, LinkedIn, Twitter, etc. A wide selection of journals (inclusive of 9 subjects, more than 200 journals)

Providing 24-hour high-quality service

User-friendly online submission system

Fair and swift peer-review system

Efficient typesetting and proofreading procedure

Display of the result of downloads and visits, as well as the number of cited articles

Maximum dissemination of your research work

Submit your manuscript at: http://papersubmission.scirp.org/

Or contact am@scirp.org 